\title{
A Cable Driven Parallel Robot with a Modular End Effector for the Installation of Curtain Wall Modules
}

\author{
K. Iturralde a M. Feucht ${ }^{\mathrm{a}}$, R. Hu ${ }^{\mathrm{a}}$, W. Pan ${ }^{\mathrm{a}}$, M. Schlandt ${ }^{\mathrm{a}}$, T. Linner ${ }^{\mathrm{a}}$, T. Bock ${ }^{\mathrm{a}}$, \\ J.-B. Izard ${ }^{\mathrm{b}}$, I. Eskudero ${ }^{\mathrm{b}}$, M. Rodriguez ${ }^{\mathrm{b}}$, J. Gorrotxategi ${ }^{\mathrm{b}}$, J. Astudillo ${ }^{\mathrm{b}}$, J. Cavalcanti ${ }^{\mathrm{c}}$, \\ M. Gouttefarde ${ }^{c}$, M. Fabritius ${ }^{\mathrm{d}}$, C. Martin ${ }^{\mathrm{d}}$, T. Henninge ${ }^{\mathrm{e}}$, S. M. Normes ${ }^{\mathrm{e}}$, Y. Jacobsen ${ }^{\mathrm{e}}$, \\ A. Pracuccif , J. Cañada ${ }^{g}$, J.D. Jimenez-Vicaria ${ }^{\text {h }}$, C. Paulotto ${ }^{\text {, }}$ R. Alonso ${ }^{i}$, L. Elia ${ }^{i}$ \\ ${ }^{a}$ Chair of Building Realization and Robotics, Technical University of Munich, Germany \\ ${ }^{b}$ TECNALIA, Basque Research and Technology Alliance (BRTA), Spain \\ ${ }^{c}$ LIRMM, CNRS, France ${ }^{d}$ IPA, Fraunhofer, Germany \\ ${ }^{e}$ nLink, Norway ${ }^{f}$ Focchi Spa, Italy ${ }^{g}$ Vicinay-Cemvisa, Spain \\ ${ }^{h}$ Acciona, Spain $\quad{ }^{i}$ R2M Solution, United Kingdom \\ E-mail: kepa.iturralde@br2.ar.tum.de
}

\begin{abstract}
-
The installation of curtain wall modules (CWMs) is a risky activity carried out in the heights and often under unfavorable weather conditions. CWMs are heavy prefabricated walls that are lifted normally with bindings and cranes. High stability is needed while positioning in order not to damage the fragile CWMs. Moreover, this activity requires high precision while positioning brackets, the modules, and for that reason, intensive survey and marking are necessary. In order to avoid such inconveniences, there were experiences to install façade modules in automatic mode using robotic devices. In the research project HEPHAESTUS, a novel system has been developed in order to install CWMs automatically. The system consists of two sub-systems: a cable driven parallel robot (CDPR) and a set of robotic tools named as Modular End Effector (MEE). The platform of the CDPR hosts the MEE. This MEE performs the necessary tasks of installing the curtain wall modules. There are two main tasks that the CDPR and MEE need to achieve: first is the fixation of the brackets onto the concrete slab, and second is the picking and placing of the CWMs onto the brackets. The first integration of the aforementioned system was carried out in a controlled environment that resembled a building structure. The results of this first test show that there are minor deviations when positioning the CDPR platform. In future steps, the deviations will be compensated by the tools of the MEE and the installation of the CWM will be carried out with the required accuracy automatically.
\end{abstract}

\section{Keywords -}

Automation; On-site; Robotics; Facade

\section{$1 \quad$ Introduction}

The European construction sector constitutes an immense market. It is one of the main industrial employers in the European Union, contributing about $9 \%$ of its GDP, with an annual turnover of more than $€ 1,500$ million and a direct workforce of 18 million people [1]. Despite the fact that the construction sector is a fairly traditional sector, trends such as smart construction, involving advanced materials, innovative processes and concepts and green approaches, are becoming more noticeable.

The curtain wall modules (CWMs) are the building envelope technological system which represents the boundary condition between indoor and outdoor environment with the goal to guarantee and preserve the designed building performances. For this purpose, the asbuilt façade needs to guarantee the correct installation of the CWMs to achieve the performance assessed by project specs detailed in the design phase and validated with tests conducted under EN 13830. This critical but fundamental moment of installation phase requires a full accomplishment of operative instructions to guarantee the performance achievement with a strict accuracy of its component installation. Indeed, because the CWM setting is a millimetric activity due to the absolute position of façade, the installation process and regulations guarantee that the as-built façade corresponds to the design. For this reason, even if some mechanical regulations are possible through specific façade's components (bolts, screws, anchors), installers today have a central role. In addition to the installation operations to guarantee the correct setting of the CWM in line with project specs, other relevant issues related to site activities need to be managed such as risk control, 
preservation of the safety of personnel involved, and correct maintenance of the equipment used. The safety of personnel involved in all site activities (not only the one responsible for façade) is the most crucial aspect. The safety procedures are independent of specific building components, but related to general principles to be pursued for each activity during site operations based on national and local norms. In this frame, façade related risks (e.g., lifting materials, equipment placement, exclusion zones, falling restrain for personnel and material, weather condition during lifting operations) are some of the risks to be considered during CWM installation to preserve the safety operation of the site activities. In this scenario, to pursue the quality of installation while reducing its risk to preserve the site personnel's safety, automation through robot is an opportunity worth being investigated.

In order to cope with these issues, different robots for installing, painting, cleaning, delaminating, maintaining and inspecting any kind of facade were developed in the past. More specifically, several robotic devices have been classified for façade module installation [2]. Besides these single task robots, on-site factories like ABCS [3] and SMART [4, 5] developed techniques for installing fully prefabricated façade modules during the erection of new buildings. Apart from façade modules, there were experiences in on-site assembly of walls like in the Rocco project, in this case, for assembling building blocks [6]. Lee et al. [7] developed a robot on top of a platform that helps the human operator to handle a CWM. The most recent instance of the installation of a façade module with a robot dates to a manually operated robotic crane [8]. Test results show that in worst case the achieved repeatability of handler end-effector positioning is 7.0 $\mathrm{mm}$. This result might not be sufficient for the installation of CWMs. Regarding the cable robots for installing façade elements, a tendon suspended platform robot was envisioned [9], but the definition degree of that solution did not show further detail, especially regarding the necessary cranes to support the loads and forces of the cables. Moreover, that solution did not show any type of on-board tools.

Cable-driven parallel robots (CDPR) are a subclass of parallel robots [10]. Instead of rigid links, they use cables to manipulate a mobile platform. The principle is to drive a mobile element in up to 6 degrees of freedom (DOF) by attaching cables to the mobile element and by synchronously controlling their length from a base frame with winches. At least 6 cables are required for controlling all 6 DOFs of the load, while often no more than 8 cables are used for better performance. The most well-known example of such robots is aerial cameras for stadiums [11] working with 3 DOF and 4 cables, and the first concept for manipulating all DOFs of a load dates back to the 1990s [12]. Today, they have already proven their benefits, in particular for large scale industrial applications [13, 14, 15]; indeed, the principle of a CDPR can be adapted to move heavy payloads over large dimensions. For the same reasons, CDPRs have being theorized in the past for several construction applications, from manipulating elements, contour crafting, to building inspection $[9,16]$.

In the HEPHAESTUS project, a redundantly constrained cable robot was built. The redundancy of using eight cables to control the six degrees of freedom of the platform increases the available workspace volume. Only few related works involving cable robots in the field of construction can be found. In [17], a concept for a cable robot for large-scale assembly of solar power plants is introduced. In [18], a cable robot concept for a contour crafting system is described. In [19, 20], cable-robots for automated brick laying can be found.

The work performed within the HEPHAESTUS project [21] features for the first time that a CDPR is designed, built and deployed specifically for the construction sector, with the primary purpose of installing CWMs, which encompasses two main tasks: bracket installation and module installation. The advantages of cable robots in HEPHAESTUS are their large workspace, high payloads, reconfigurability and modular components, which make it easily transportable.

\section{Concept description}

The aforementioned tasks (bracket installation and module placement) require high relative and absolute accuracy. To accomplish such accuracy, it is necessary to foresee the precision of the CDPR, which was estimated to have a tolerance of $40 \mathrm{~mm}$ [22] in previous phases. Therefore, in previous stages of the project, it was foreseen that there would be two means for installing the CWM: the CDPR for the rough positioning and the Modular End Effector (MEE) along with its tools for the fine positioning.

\section{$2.1 \quad$ CDPR}

From a geometrical point of view, a CDPR is an association of cables of variable lengths linking a drawing point attached to base frame, and a fixing point attached to the mobile element or platform. How these drawing and fixing points are positioned in space, respectively in the general frame and the mobile platform frame, and how they are connected together formulate a configuration.

\subsubsection{CDPR calculation}

The geometrical design of the CDPR presented in Figure 1 can be summarized as the definition of the following parameters: (i) number of cables, (ii) geometry of the structure, (iii) geometry of the platform, and (iv) cable configuration. Based on previous studies indicating 
that CDPRs driven by eight cables have appropriate performances [23], this number of cables was chosen. The parameters (ii) and (iii) are defined by the positions of the drawing points and attachment points respectively (see Figure 1). The cable configuration (iv) defines the pairs of drawing and attachment points that are connected by cables. Therefore, significant efforts in the design of this CDPR were dedicated to the definition of an appropriate set of parameters (ii), (iii) and (iv).

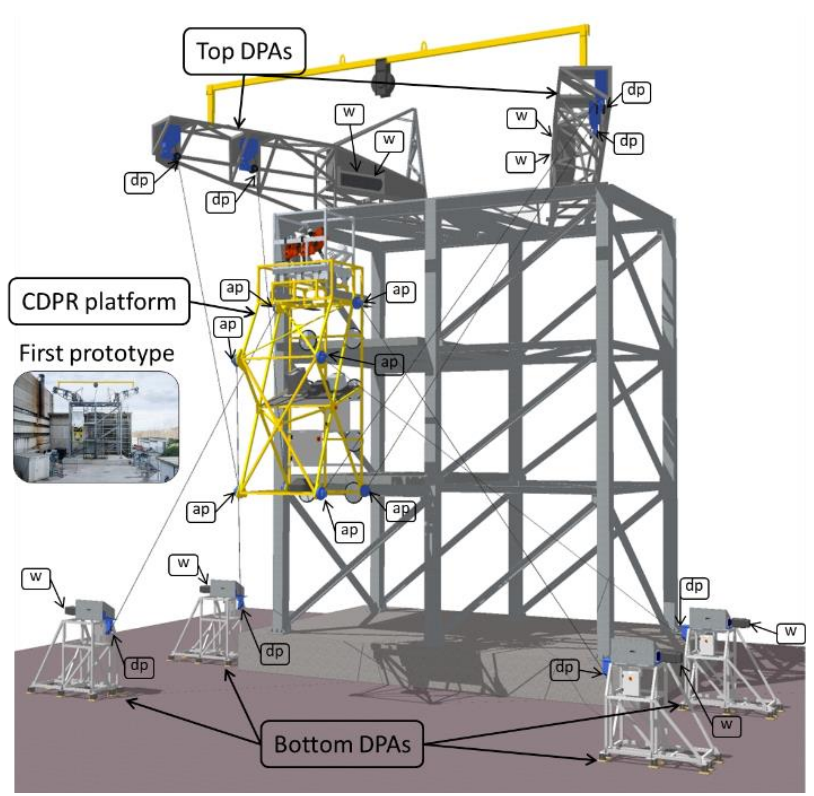

Figure 1. Hephaestus CDPR prototype

The abstract goal of finding an appropriate set of parameters was formulated as an optimization problem. The cost function of the proposed optimization problem is the maximal cable tension, directly linked to the Safe Working Load (SWL), obtained during operation across the building facade. The choice of this cost function is motivated by the direct relationship between the SWL and the cost of the machine. Minimizing the SWL leads to minimizing the maximal loads that are applied on the mechanical parts of the CDPR and, therefore, the cost is minimized. In addition, the constraints of the optimization problem include the positioning accuracy which should meet the precision necessary for the installation of the CWMs. Further details on the geometrical optimization of the Hephaestus CDPR prototype can be found in [24].

\subsubsection{CDPR hardware}

The Hephaestus CDPR is composed of 7 subassemblies. The first set of subassemblies provides the means of controlling the lengths of the cables. These subassemblies are fixed to the building, which works as the base frame for the robot. They are called drawing point assemblies (DPA in Figure 1) and come in two types. The first type is fixed at ground level, materializing the lower drawing points (dp in Figure 1) of the proposed configuration (one per assembly). The second type is attached to the building top slab. Each top DPA materializes two among the top drawing points. There are, therefore, two top DPAs and 4 bottom DPAs (see Figure 1).

Each drawing point need a winch, a swivel pulley at the location of the drawing point, and a force sensor for monitoring the cable tension. The components are the same for all drawing points. The travelling sheave winches (VICINAY winches WB21.L30S.1: SWL $15.7 \mathrm{kN}$, drum torque $2128 \mathrm{Nm}$, velocity $30 \mathrm{~m} / \mathrm{min}$, cable travel 16m, see w in Figure 1 and Figure 2) are powered by a servomotor with brake and absolute multi-turn encoder integrated, associated to a gearbox and wire rope spooling mechanism synchronized with the grooved drum.

The swivel pulley installed at the theoretical location of the drawing point rotates around a vertical axis; it guides the cable towards the matching fixing point. The force sensor is embedded in the shaft of the sheave directing the cable from the winch to the swivel pulley. The steel wire rope is a $\emptyset 11 \mathrm{~mm}$ non-rotating cable with a minimum breaking load of $115.5 \mathrm{kN}$.

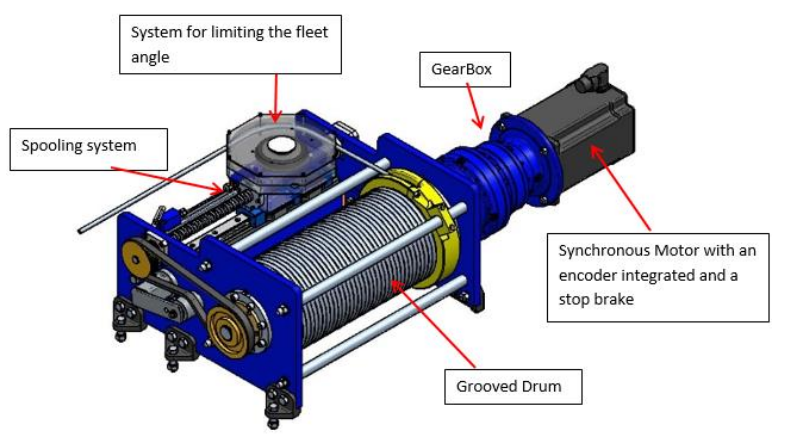

Figure 2. CAD view of the VICINAY Winch WB21.L.30S.1

The mechanical structure of the DPAs is designed in order to transfer the load from the swivel pulleys and the winches to the anchoring elements. They were designed to show a displacement of less than $50 \mathrm{~mm}$ at the drawing point location when loaded with the winches' SWL. Steel anchorage plates are embedded during the construction of the concrete building in the third (top) slab. The supporting structures are later welded to these anchorage plates in the correct position so that the DPA are in the correct coordinates with the required tolerances, with the drawing point positions being monitored continuously by a surveyor with a total station

Another CDPR subassembly is the platform (see Figure 1 and Figure 5). It features the 8 fixing points 
placed accordingly to the dimensions set in the configuration, as well as the various tools and power systems for the MEE. The total weight of the fully loaded platform reaches $1460 \mathrm{~kg}$, in which $350 \mathrm{~kg}$ accounts for the carried CWM.

The norms applied during the design are ISO 4301, ISO 16625 and FEM 1.001. All elements have been designed with a safety factor of at least 5.6 in order to match the M5 mechanism group requirements.

The final CDPR subassembly is a weatherproof electrical cabinet housing the central control unit. It features the servomotor drives, the associated power units, the central PLC where the central control is implemented, and the associated inputs and outputs acquisition system. The cables towards the platform (data and power) are directed to it by means of a cable chain mechanism fixed to a beam installed between the two top DPAs.

\subsection{MEE and its components}

The MEE is the set of tools that performs each of the activities necessary for installing the CWM onto the structure of the building. The MEE is fixed to the CDPR platform (see Figure 5). In the case of the HEPHAESTUS project, two main activities need to be performed. First, there is the fixation of the bracket onto the concrete slab. This task is achieved by a robotic arm. Second, there is a placement of the CWM modules onto the brackets. This task is achieved by a vacuum system attached to the CDPR platform that picks a CWM from an inclined magazine and releases the CWM when it is placed onto the brackets.

\subsubsection{Robotic arm and its tools}

Selected tools need to be manipulated by the robot in order to mount brackets to hold the CWM to the building. The most versatile method is in-situ mounting and this was the chosen approach in this project. The list of actions needed to be handled by the robot is concluded: drilling of holes for anchor bolts, picking and placement of bracket over holes, picking and placement of anchor bolts in holes, setting of bolts into holes, and tightening of anchor bolts nuts to set torque. A Universal Robots UR10e was selected as the tool manipulator. This was done based on previous experience with this robot and its possibilities and limitations, specifically regarding drilling in concrete. The robot arm also allows for excellent adaptability to changes based on underway project learnings. The arm was mounted on a custom structure made of profiled aluminum bars. A toolchanger system was integrated to give the robotic arm the possibility to manipulate a variety of tools.

Four tools were put together to achieve the needed customized functionality: 1) the drilling tool, 2) the bracket picker and holder, 3) the setting tool with a hammer function, and 4) a tool to torque the nut of the anchor.

The cycle is completed by the robotic arm returning to the bracket holder, and releasing the vacuum and magnets from the slab and bracket correspondingly, before the bracket holder is returned to the tool dock. ${ }^{\wedge}$

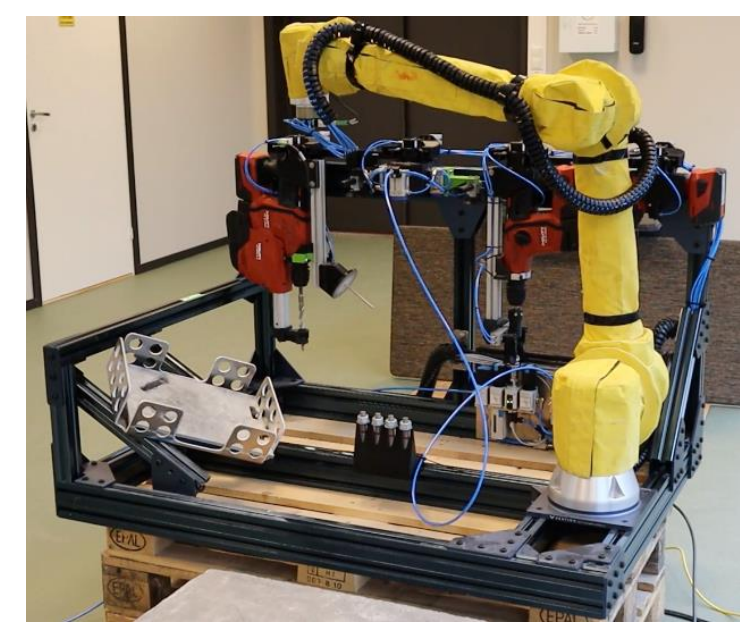

Figure 3. Robotic arm and its tools before mounting on the CDPR platform.

\subsubsection{Stabilizer of the robot's frame}

One of the issues regarding the accuracy of the robotic arm relied on the stability of the frame that hosts the robotic arm and its tools while performing tasks.

For achieving such needs, a linear system with vacuum cups was defined, tested and prototyped. This linear system was conceived for hosting forces of up to $1500 \mathrm{~N}$.

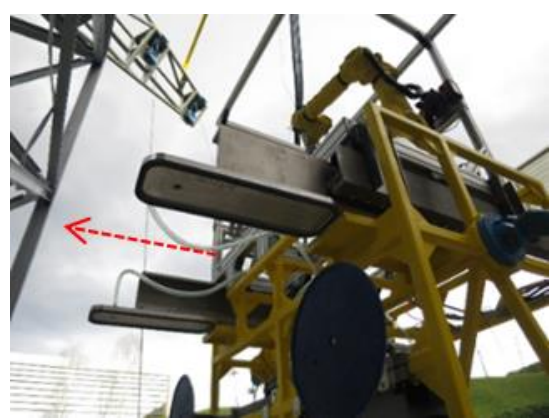

Figure 4. Stabilizer of the robot's frame prototype during the opening of the stabilizers.

The linear system consisted on two subsystems: the linear actuators and the machined steel profiles (see Figure 4) that run along the rails with the help of carriers.

\subsubsection{Vacuum Lifting System for picking and placing the CWM}

The Vacuum Lifting System (VLS) is capable for 
picking and placing the CWM of $350 \mathrm{~kg}$ during operations that require inclined plans.

The VLS is designed to grip, in vertical position, a CWM of the aforementioned mass, with a smooth glass surface, and a surface $A_{z x}$ of $5.1 \mathrm{~m}^{2}$. The CWM is a parallelepiped with three different faces $A\left(A_{y z}, A_{z x}, A_{x y}\right)$ perpendicular to $x, y, z$ axes with values $A=\left(\begin{array}{lll}0.68 & 5.1 & 0.3\end{array}\right)^{T} m^{2}$ and showing a maximum aerodynamic coefficient $c_{a}$ at 1.32 . It would be possible to work in both dry and wet states, without ice, with the friction coefficient being estimated $0.2(\mu$ in (3)). The VLS is dimensioned to lift a load greater than or equal to twice its design load with the minimum relative vacuum pressure $q_{r}$. Finally, the altitude should be at least $900 \mathrm{~m}$ from sea level, the temperature between -5 to $40^{\circ} \mathrm{C}$, and accordingly the wind pressure $q_{w}$ during service is estimated lower than $125 \mathrm{~N} / \mathrm{m}^{2}$ and the vacuum differential pressure $q_{r}$ at least equal to $600 \mathrm{mbar}$. The system creates a grip force $f_{g}$ between the surfaces of the CWM and those of the $n=8$ suction cups, showing a diameter $d$ of $\varnothing 360 \mathrm{~mm}$. The total load solicitation vector $\boldsymbol{s}$ is the sum of: the CWM mass $m$ multiplied by gravity vector $g$, and by acceleration $j=\left(\begin{array}{lll}1 & 1 & 1\end{array}\right) \mathrm{m} / \mathrm{s}^{2}$ due to the movement, and the forces due to the wind action $f_{w}$, each factorized with the applicable partial safety coefficients $\left(\gamma_{p}=1.1\right)$, which are expressed as follows:

$$
\begin{gathered}
f_{g}=n \frac{\pi \cdot d^{2} \cdot q_{r}}{4}=48.86 k N \\
f_{w}=c_{a} \cdot q_{w} A=\left(\begin{array}{lll}
112 & 841 & 50
\end{array}\right)^{T} N \\
s=\left(\frac{m(g+j)}{\mu \cdot\left(\begin{array}{lll}
1 & 1 & 1
\end{array}\right)^{T}}+\frac{\gamma_{p} \cdot f_{w}}{\left(\begin{array}{lll}
\mu & 1 & \mu
\end{array}\right)^{T}}\right)=\left(\begin{array}{l}
2.52 \\
2.52 \\
20.8
\end{array}\right) k N
\end{gathered}
$$

The current VLS design is validated by $f_{g}$ being greater than twice any component of $\boldsymbol{s}$.

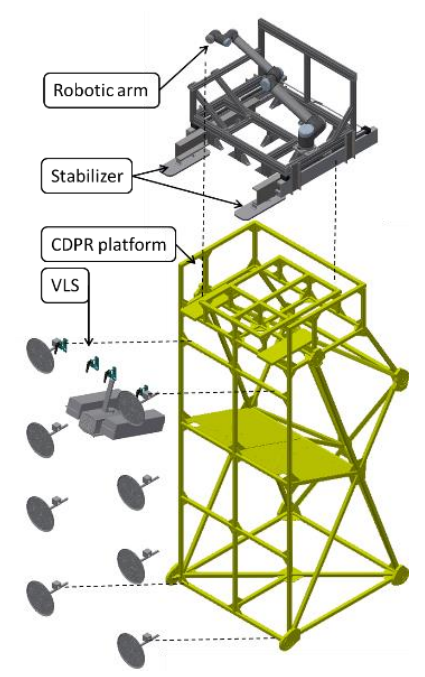

Figure 5. location of the MEE on the platform.
The VLS, and its warnings and safety measures are connected to the Beckhoff control and therefore it can be activated automatically as explained in the next section.

\subsection{Control system}

In Figure 6, the scheme of the hardware and wiring of the HEPHAESTUS robot is shown. The system consists of 4 PCs in total. Starting from the left side in the scheme, a standard PC is used to execute a software tool to automate the façade panel installation. This tool commands the steps in the correct order to mount the facade modules. Furthermore, it provides a GUI for the operator to control the whole HEPHAESTUS robot. It is connected to a total station via TCP/IP, which can measure the absolute pose of the cable robot platform and to the IPC on which the cable robot controller is running. The cable robot controller is based on the TwinCAT 3 software from BECKHOFF [25]. It consists of a softPLC and a motion controller. The latter can either be a Beckhoff CNC, or an advanced motion controller. The IPC is connected via WLAN (CANopen) to the Radio Control, via Ethernet (EtherCAT) to the safety sensors, I/Os, force sensors and drives.

Furthermore, the IPC has an Ethernet (EtherCAT) interface to the IPC of the MEE, which is integrated within the EtherCAT network as an EtherCAT slave. On the MEE IPC a PLC is implemented to control the MEE system consisting of the ROS-PC to control the URRobot, the stabilizer, and the vacuum system.

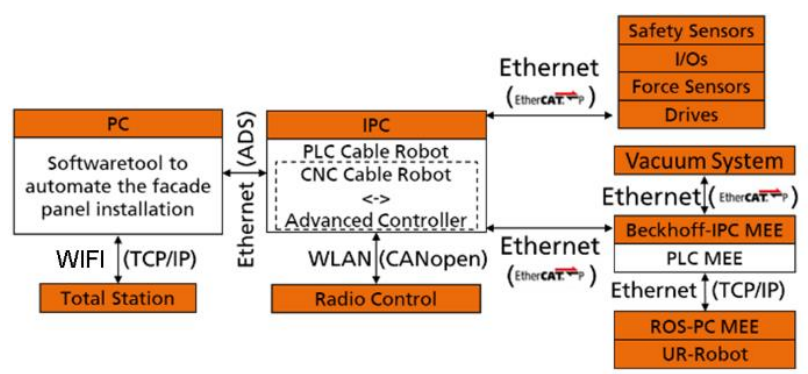

Figure 6. Scheme of the hardware and wiring of the HEPHAESTUS cable robot.

The main application controls the interactions between the user and the main controller. The application UI shows cable robot data, such as cable tensions, and each state the robot is performing in real time. It also allows the user to intercept each state, pausing the operation, or to stop the task. It is connected to the cable robot controller, allowing the user to move the cable robot and see the state the cable robot and the MEE are in at any moment, allowing the user to operate and control it, and the total station controller, allowing the user to obtain position and rotation measures at will. 
A precise kinematic model is necessary in order to guarantee a satisfying positioning accuracy of the CDPR. Since the pose of the platform is computed based on the winch motor positions, cable sagging and elongation may be considered [26]. Among other aspects related to the position tracking control for CDPRs, these are still ongoing research subjects addressed in this project.

\subsection{State Machine}

The main controller is designed to operate as a state machine that controls all the individual controllers. Likewise, it is designed to work separately from the UI, merging the real time environment with the UI thread, and it controls all the error controllers to broadcast individual error signals. There are two main operations that the robot must do in order to complete the CWM installation successfully: first drill and set the brackets in the correct positions, and then set the CWMs in the corresponding brackets. To do both of them there are several states that the controller must follow, each one of them linked to a specific controller (cable robot control, MEE control or total station control). Each state, as shown in the simplified state machine diagram (see Figure 7), has an optional breakpoint where the user can stop the operation if a malfunction is detected. Besides these two main operations, the state machine contains also the semi-automatic initialization states of the total station.

\section{$3 \quad$ Prototyping and tests}

The first demonstration tests were performed in TECNALIA facilities in Derio, Basque Country (Spain). Once all the components of the demonstrator were installed, the operation of all the components (motors, movement of the robot, positioning in relation with the steel structure, sensor, etc.) was verified. This was the first time the different elements of the robot (winches with cable pulling on the platform/base) and the higherlevel control of the robot that makes the coordination of the winches were put together.

\subsection{Building structure used for the demonstration}

For this purpose, a steel structure has been erected matching the foreseen dimensions of the demonstration building: $10.2 \mathrm{~m}$ high, $8.80 \mathrm{~m}$ wide, and $2.7 \mathrm{~m}$ deep. Two concrete slabs have been installed at the first and second floor to perform all tests required for installing one CWM.

The steel structure has features to accommodate the top DPAs on the top floor; the bottom DPAs are directly anchored to the ground (Figure 1).

The higher platform empty weight more than expected and the SWL lower than originally planned (respectively $1110 \mathrm{~kg}$ instead of 910 and $15.7 \mathrm{kN}$ instead of 20) led to the nominal transit positions of the top row of panels not being accessible. The transit distance for the top floor panels therefore needed to be reduced from 600 to $450 \mathrm{~mm}$.

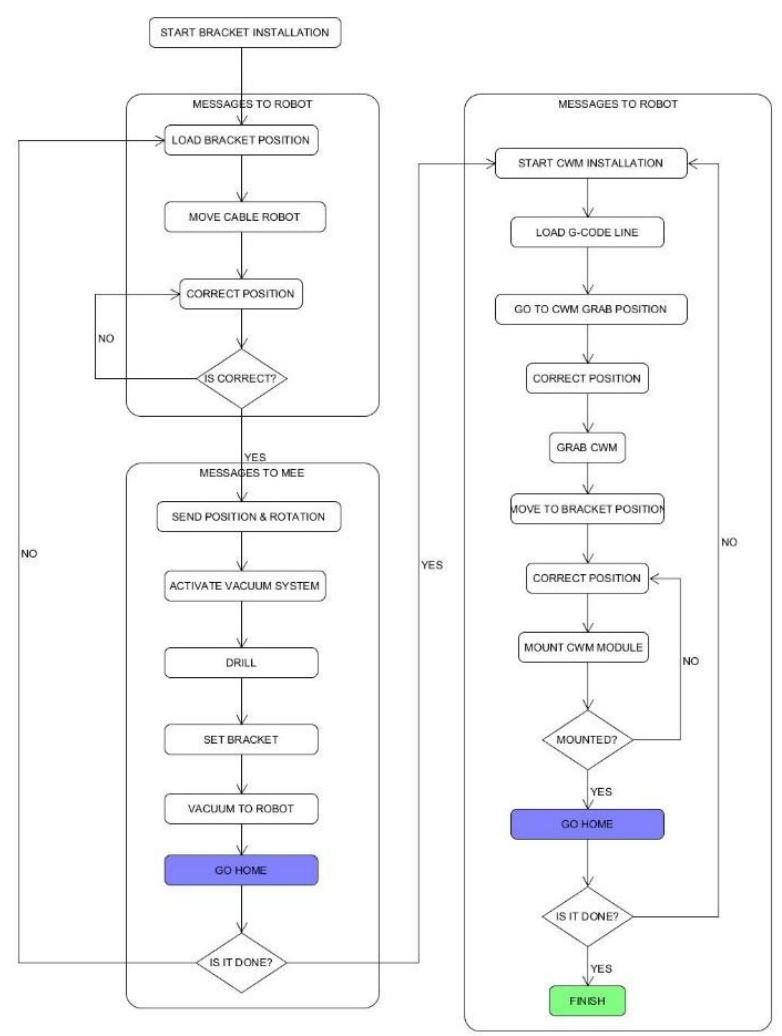

Figure 7. Simplified state machine diagram

\subsection{Installation of the CDPR}

After the erection of the building, the DPAs, mobile platform and control cabinet were brought to the building site. The top DPAs (2500kg each) were installed on the building top floor by the means of a mobile crane. The bottom DPAs (1100kg each) can be moved around using a forklift. Once the DPAs have been installed, calibration must be carried out.

Calibration of the drawing point positions is performed thanks to the integration of total station targets onto the swivel pulley assemblies. Each swivel pulley assembly features 4 targets; their positions are used to build a local frame to reconstruct the current position of the associated drawing point. In order to calibrate the full system, apart from the A and B points, there are 3 Leica $360^{\circ}$ targets [27] attached to the cable robot platform in order to track it on the move, 3 Leica $20 \times 20 \mathrm{~mm}$ reflectors attached to the cable robot platform in order to calibrate the origin point of the MEE with respect to the cable robot platform frame, and at least 3 Leica $20 \times 20$ 
$\mathrm{mm}$ reflectors to triangulate the building from the total station. It is highly advisable to calibrate all the prism and reflectors at the same time to achieve best possible accuracy.

The calibration procedure has been performed at the same time as the installation of the DPAs, with the drawing point positions being monitored continuously by a surveyor with a total station. The objective was to have the DPAs installed as close as possible to their theoretical positions: the distance to the theoretical positions was measured at maximum $19 \mathrm{~mm}$.

\subsection{Results}

The first results of the demonstration show a better performance than expected in previous phases of the research project (see Figure 8 and check video in [21]). The maximum position error of the CDPR is about $20 \mathrm{~mm}$ and the max orientation error about $0.8 \mathrm{deg}$. Moreover, the preliminary results show a promising repeatability (with an accuracy of 1-2 mm) of the CDPR while moving the platform within the workspace. However, more tests are necessary to define better this parameter. The deviations in respect to the desired position were supposed to be adjusted by the MEE while fixing the brackets. However, due to time constrains during the installation of the CDPR and the MEE, some calibration issues appeared and the transformed of the MEE with regard to the $0,0,0$ point of the building was not achieved properly. For that reason, some deviations occurred during the placement of the bracket. This is a topic that will be improved in the next phase.
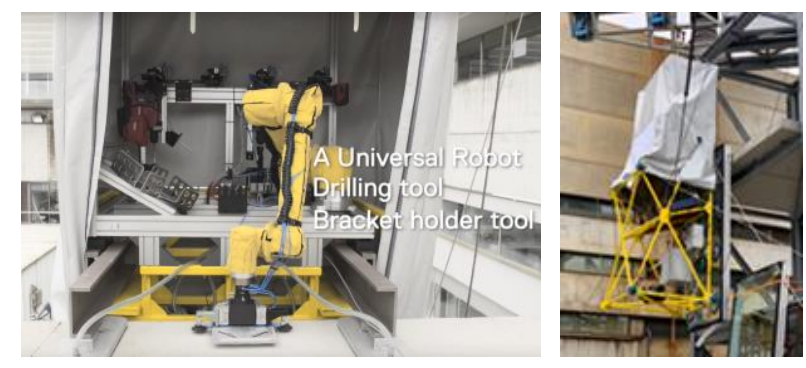

Figure 8. MEE and CDPR in operation as in [21]

\section{$4 \quad$ Conclusions and future work}

The first test of a CDPR for installing CWMs was achieved with better than expected results. However, there are still some points that need to be improved:

- Improve the calibration of the MEE in regards with the building in order to achieve a better accuracy.

- The detection of the CWM while it stands on the magazine and measuring its location.

- Detection of the brackets that are already fixed on the building slab in order to adjust, if necessary, the
CDPR path while placing the CWM.

In order to seek for future commercialization, a market research was carried out which found a growing awareness from building owners and residents about comfort and health as well as political and economic drivers (e.g: nZEB and other EU directives, incentive schemes and favorable tax regimes, especially for green construction). Technological innovations will complete these drivers, making investors, policymakers and professionals (i.e. architects, designers as well as façade manufacturers) accelerate the adoption of construction robots. Therefore, the goal is that in the coming years the innovations mentioned in this paper will reach the market with the following exploitable results: i) CDPR for vertical works: suitable for handling, moving and placing CWMs; ii) MEE: including several tools to automate the insertion of a connector onto the building's structure; iii) curtain wall adapted to robotic installation: for fixing elements of the CWM to slab; CWM to bracket; and connection between CWMs; and iv) Hephaestus system: as an integrated solution for handling and installing CWMs. To facilitate commercialization of new device categories, standards can do the following:

1. Standardize the components and interfaces from which it is made in order to allow for faster development and efficient supply chains ("interoperability").

2. Standardize the processes and infrastructures surrounding the new technology or product/service.

3. Ensure quality and efficiency of the technology and/or its development processes in order to minimize the risks for the involved stakeholders.

During a final demonstration stage of the project, the robot will complete the installation of a set of CWMs covering part of the façade of a demo building particularly built and enabled for these activities. This demo building has been erected in the machinery park owned by ACCIONA and is located in Noblejas, Toledo (Spain), so that the performance of the cable robot can be demonstrated in a real construction environment. The emo building was erected with three floors and a total height of $10.2 \mathrm{~m}$, and the façade is $8.5 \mathrm{~m}$ wide. To access the various floors of the demo building during demonstration activities, a staircase has been installed on the back side of the building where no facade panels will be installed. The Hephaestus system will be validated, among other performance indicators, in terms of time required to complete the operations for the CWM placing, the accuracy, the efficiency and the usability for workers of the construction sector. Also, special care will be taken in order to fulfil the safety requirements and recommendations for these robotic operations. 


\section{Acknowledgements}

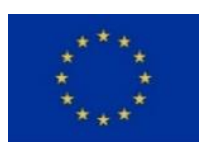

This project has received funding from the European Union's Horizon 2020 research and innovation programme under grant agreement No 732513.

\section{References}

[1] European Commission, "Construction. Internal Market, Industry, Entrepreneurship and SMEs," [Online]. Available: https://ec.europa.eu/growth/sectors/construction_en [Accessed 10 June 2020].

[2] Bock T. and Iturralde K., "Automated and Robotic Process Lifecycle of Prefabricated Facades," in Neue Entwicklungen im Betonbau, Beuth Verlag GmnH, 2019, pp. 117-129.

[3] Obayashi-Corporation, Director, ABCS Riverside Sumida Bachelor Dormitory Documentary Video. [Film]. 1993.

[4] Miyakawa H., Ochiai J., Oohata K., and Shiokawa T., "Application of automated building construction system for high rise office building," in Proceedings of 17th International Symposium on Automation and Robotics in Construction, Taipei, Taiwan, 2000.

[5] Maeda J., "Development and Application of the SMART System," in Automation and Robotics in Construction, Elsevier Science B.V., 1994, pp. 457 464.

[6] Gambao E., Balaguer C., Barrientos A., Saltaren R., and Puente E., "Robot assembly system for the construction process automation," in Proceedings of International Conference on Robotics and Automation, Albuquerque, USA, 1997.

[7] Lee S.Y., Gil M., Lee K., Lee S and Han C., "Design of a ceiling glass installation robot," in Proceedings of 24th International Symposium on Automation and Robotics in Construction, Madras, 2007.

[8] Činkelj J., Kamnik R., Čepon P., Mihelj M. and Munih M., "Closed-loop control of hydraulic telescopic handler.," Automation in Construction, 19(7), pp. 954 963., 2010.

[9] Thompson C. and Campbell P.J., "Tendon suspended platform robot". U.S. Patent Patent 5,585,707, 17 December 1996.

[10] Cone L., "Skycam - an aerial robotic camera system", Byte, vol. 10, no. 10, pp. 122-132, 1985.

[11] Albus J., Bostelman R. and Dagalakis N., "The NIST Robocrane," Journal of Robotic Systems, vol. 10, no. 5, pp. 702-724, 1993

[12] Pott A., Cable-driven parallel robots: theory and application, Springer, 2018.

[13] Gouttefarde M., Collard J.-F., Riehl N. and Baradat C., "Geometry selection of a redundantly actuated cablesuspended parallel robot," IEEE Transactions on Robotics, vol. 31, no. 2, pp. 501-510, 2015.

[14] Pott A., Mütherich H., Kraus W., Schmidt V., Miermeister P. and Verl A., "IPAnema: A family of
Cable-Driven Parallel Robots for Industrial Applications," Cable-Driven Parallel Robots, 2013.

[15] Culla D., Gorrotxategi J., Rodríguez M., Izard J.-B. and Hervé P.-E., "Full Production Plant Automation in Industry Using Cable Robotics with High Load Capacities and Position Accuracy," Iberian Robotics conference, pp. 3-14, 2017.

[16] Izard J.-B., Gouttefarde M., Baradat C., Culla D. and Sallé D., "Integration of a parallel cable-driven robot on an existing building façade, " Cable-Driven Parallel Robots, pp. 149-164, 2013.

[17] Pott A., Meyer C. and Verl A., "Large-scale assembly of solar power plants with parallel cable robots," in (41st International Symposium on Robotics) and ROBOTIK 2010 (6th German Conference on Robotics) VDE, 2010.

[18] Bosscher P., Williams II R. L., Bryson L. S. and Castro-Lacouture D., "Cable-suspended robotic contour crafting system," Automation in Construction, pp. 45-55, 2007.

[19] Vukorep I., "Autonomous big-scale additive manufacturing using cable-driven robots," in Proceedings of the International Symposium on Automation and Robotics in Construction, Taiwan, 2017.

[20] Bruckmann T., Reichert C., Meik M., Lemmen P., Spengler A., Mattern H. and König M., "Concept Studies of Automated Construction Using CableDriven Parallel Robots," Cable-Driven Parallel Robots Springer, Cham, pp. 364-375, 2018.

[21] R2M Solutions, "Hephaestus - EU H2020 Project," 2017. [Online]. Available: http://www.hephaestusproject.eu/ [Accessed 10 June 2020].

[22] Taghavi M., Iturralde K. and Bock T., "Cable-driven parallel robot for curtain wall modules automatic installation," in 35th International Symposium on Automation and Robotics in Construction , Berlin, 2018.

[23] Gouttefarde M., Lamaury J., Reichert C. and Bruckmann T., "A Versatile Tension Distribution Algorithm for n-DOF Parallel Robots Driven by $\mathrm{n}+2$ Cables," IEEE Trans. Robot., vol. 31, no. 6, p. 14441457, 2015.

[24] Hussein H., Santos J.C. and Gouttefarde M., "Geometric Optimization of a Large Scale CDPR Operating on a Building Facade," in IEEE International Conference on Intelligent Robots and Systems, 2018.

[25] Beckhoff. [Online]. Available: https://www.beckhoff.de/default.asp?twincat/twincat3.htm [Accessed 09 June 2020].

[26] Izard, J. B. et al., "On the improvements of a cabledriven parallel robot for achieving additive manufacturing for construction”, Cable-Driven Parallel Robots, Springer, pp. 353-363, 2018.

[27] Leica. [Online]. Available: http://www.surveyequipment.com/PDFs/leica-whitepaper-surveying-prisms.pdf [Accessed 09 June 2020]. 